\title{
Fractional cross intersecting families
}

\author{
Rogers Mathew ${ }^{1}$, Ritabrata Ray ${ }^{2}$, and Shashank Srivastava ${ }^{3}$ \\ ${ }^{1}$ Department of Computer Science and Engineering, \\ Indian Institute of Technology Kharagpur, Kharagpur 721302, India, \\ rogersmathew@gmail.com \\ ${ }^{2}$ Department of Electronics and Electrical Communication Engineering, \\ Indian Institute of Technology Kharagpur, Kharagpur 721302, India, \\ rayritabrata96@gmail.com \\ ${ }^{3}$ Toyota Technological Institute at Chicago, Chicago 60615, USA, \\ shashanksri47@gmail.com
}

\begin{abstract}
Let $\mathcal{A}=\left\{A_{1}, \ldots, A_{p}\right\}$ and $\mathcal{B}=\left\{B_{1}, \ldots, B_{q}\right\}$ be two families of subsets of $[n]$ such that for every $i \in[p]$ and $j \in[q],\left|A_{i} \cap B_{j}\right|=\frac{c}{d}\left|B_{j}\right|$, where $\frac{c}{d} \in[0,1]$ is an irreducible fraction. We call such families $\frac{c}{d}$-cross intersecting families. In this paper, we find a tight upper bound for the product $|\mathcal{A}||\mathcal{B}|$ and characterize the cases when this bound is achieved for $\frac{c}{d}=\frac{1}{2}$. Also, we find a tight upper bound on $|\mathcal{A}||\mathcal{B}|$ when $\mathcal{B}$ is $k$-uniform and characterize, for all $\frac{c}{d}$, the cases when this bound is achieved.
\end{abstract}

\section{Introduction}

Let $[n]$ denote $\{1, \ldots, n\}$ and let $2^{[n]}$ denote the power set of $[n]$. We shall use $\left(\begin{array}{c}{[n]} \\ k\end{array}\right)$ to denote the set of all $k$-sized subsets of $[n]$. Let $\mathcal{F} \subseteq 2^{[n]}$. The family $\mathcal{F}$ is an intersecting family if every two sets in $\mathcal{F}$ intersect with each other. The famous Erdös-Ko-Rado Theorem [1] states that $|\mathcal{F}| \leq\left(\begin{array}{l}n-1 \\ k-1\end{array}\right)$ if $\mathcal{F}$ is a $k$-uniform intersecting family, where $2 k \leq n$. Several variants of the notion of intersecting families have been extensively studied in the literature. Given a set $L=\left\{l_{1}, \ldots, l_{s}\right\}$ of nonnegative integers, a family $\mathcal{F} \subseteq 2^{[n]}$ is L-intersecting if for all $F_{i}, F_{j} \in \mathcal{F}, F_{i} \neq$ $F_{j},\left|F_{i} \cap F_{j}\right| \in L$. Ray-Chaudhuri and Wilson in [2] showed that if $\mathcal{F}$ is $k$-uniform and $L$-intersecting, then $|\mathcal{F}| \leq\left(\begin{array}{l}n \\ s\end{array}\right)$ and the bound is tight. Frankl and Wilson in [3] showed a tight upper bound of $\left(\begin{array}{l}n \\ s\end{array}\right)+\left(\begin{array}{c}n \\ s-1\end{array}\right)+\cdots+\left(\begin{array}{l}n \\ 0\end{array}\right)$ if the restriction on the cardinalities of the sets of an $L$-intersecting family is relaxed. Further, if $L$ is a singleton set, then Fisher inequality [4] gives an upper bound of $|\mathcal{F}| \leq n$ for the cardinality of an $L$-intersecting family $\mathcal{F}$. Recently, in [5], Balachandran et al. introduced a fractional variant of the classical $L$-intersecting families. For a survey on intersecting families, see [6]. 
Two families $\mathcal{A}, \mathcal{B} \subseteq 2^{[n]}$ are cross-intersecting if $|A \cap B|>0, \forall A \in \mathcal{A}, B \in \mathcal{B}$. Pyber in [7] showed that if $n \geq 2 k$, and $\mathcal{A}, \mathcal{B} \subseteq\left(\begin{array}{c}{[n]} \\ k\end{array}\right)$ is a cross-intersecting pair of families, then $|\mathcal{A}||\mathcal{B}| \leq\left(\begin{array}{c}n-1 \\ k-1\end{array}\right)^{2}$. Frankl et al. in [8] showed that if $\mathcal{A}, \mathcal{B} \subset\left(\begin{array}{c}{[n]} \\ k\end{array}\right)$ such that $|A \cap B| \geq t$ for all $A \in \mathcal{A}$ and $B \in \mathcal{B}$, then for all $n \geq(t+1)(k-t+1)$, $|\mathcal{A}||\mathcal{B}| \leq\left(\begin{array}{c}n-t \\ k-t\end{array}\right)^{2}$, the cross-intersecting version of the Erdős-Ko-Rado Theorem. A cross-intersecting pair of families $\mathcal{A}, \mathcal{B} \subseteq 2^{[n]}$ is said to be $l$-cross-intersecting if $\forall A \in \mathcal{A}, B \in \mathcal{B},|A \cap B|=l$, for some positive integer $l$. Ahlswede, Cai and Zhang showed in [9], for all $n \geq 2 l$, a simple construction of an $l$-cross-intersecting pair $(\mathcal{A}, \mathcal{B})$ of families of subsets of $[n]$ with $|\mathcal{A}||\mathcal{B}|=\left(\begin{array}{c}2 l \\ l\end{array}\right) 2^{n-2 l}=\Theta\left(\frac{2^{n}}{\sqrt{l}}\right)$. Later Alon and Lubetzky in [10] showed that the $\Theta\left(\frac{2^{n}}{\sqrt{l}}\right)$ bound is tight and characterized the cases when the bound is achieved.

In this paper, we introduce a fractional variant of the $l$-cross-intersecting families. Let $\mathcal{A}=\left\{A_{1}, \ldots, A_{p}\right\}$ and $\mathcal{B}=\left\{B_{1}, \ldots, B_{q}\right\}$ be two families of subsets of $[n]$ such that for every $i \in[p]$ and $j \in[q],\left|A_{i} \cap B_{j}\right|=\frac{c}{d}\left|B_{j}\right|$, where $\frac{c}{d} \in[0,1]$ is an irreducible fraction. We call such an $(\mathcal{A}, \mathcal{B})$ pair a $\frac{c}{d}$-cross-intersecting pair of families. Given $c, d$, and $n$, let $\mathcal{M}_{\frac{c}{d}}(n)$ denote the maximum value of $|\mathcal{A}||\mathcal{B}|$ where $(\mathcal{A}, \mathcal{B})$ is a $\frac{c}{d}$-cross intersecting pair of families of subsets of $[n]$. We have the following results:

Theorem 1.1. $\mathcal{M}_{\frac{c}{d}}(n)=2^{n}$

When $\frac{c}{d}=0, \mathcal{A}=2^{[n]}, \mathcal{B}=\{\emptyset\}$ is a maximal pair. In fact, $\mathcal{A}=2^{[k]}, \mathcal{B}=\mathcal{P}(S)$, where $\mathcal{P}(S)$ is the power set of $S=\{k+1, \ldots, n\}$, are the only maximal pairs up to a relabelling of the elements, $0 \leq k \leq n$. When $\frac{c}{d}=1, \mathcal{A}=\{[n]\}$ and $\mathcal{B}=2^{[n]}$ is a maximal pair. In fact, $\mathcal{B}=2^{[k]}, \mathcal{A}=\{A: A=[k] \cup T$, where $T \in \mathcal{P}(S)\}$, where $\mathcal{P}(S)$ is the power set of $S=\{k+1, \ldots, n\}$, are the only maximal pairs up to a relabelling of the elements, $0 \leq k \leq n$. In Theorem 1.2 , we characterize all maximal pairs when $\frac{c}{d}=\frac{1}{2}$.

Theorem 1.2. Let $(\mathcal{A}, \mathcal{B})$ be a $\frac{1}{2}$-cross intersecting pair of families of subsets of $[n]$ with $|\mathcal{A}||\mathcal{B}|=2^{n}$. Then $(\mathcal{A}, \mathcal{B})$ is one of the following $\left\lfloor\frac{n}{2}\right\rfloor+1$ pairs of families $\left(\mathcal{A}_{k}, \mathcal{B}_{k}\right), 0 \leq k \leq\left\lfloor\frac{n}{2}\right\rfloor$, up to isomorphism.

$$
\begin{gathered}
\qquad \mathcal{A}_{0}=2^{[n]} \text { and } \mathcal{B}_{0}=\{\emptyset\} \\
\qquad \mathcal{A}_{k}=\left\{A \in 2^{[n]}:|A \cap\{2 i-1,2 i\}|=1 \quad \forall i, 1 \leq i \leq k\right\} \\
\mathcal{B}_{k}=\left\{B \in 2^{[n]}:|B \cap\{2 i-1,2 i\}| \in\{0,2\} \quad \forall i, 1 \leq i \leq k \text { and } \forall j>2 k, j \notin B\right\}, \\
\text { where } 1 \leq k \leq\left\lfloor\frac{n}{2}\right\rfloor .
\end{gathered}
$$


It would be interesting to show a characterization theorem for any $\frac{c}{d} \in[0,1]$. We do have such a general characterization theorem (along with a new tight upper bound) in Theorem 1.3 for the case when $\mathcal{B}$ is $k$-uniform. The proof is a direct application of Theorem 1.1 in [10].

Theorem 1.3. Let $(\mathcal{A}, \mathcal{B})$ be a $\frac{c}{d}$-cross intersecting pair of families of subsets of $[n]$. Let $\mathcal{B}$ be $k$-uniform. Then, there exists some $k_{0}>0$, such that for $k>k_{0}$ we have

$$
|\mathcal{A}||\mathcal{B}| \leq\left(\begin{array}{l}
\frac{2 c k}{d} \\
\frac{c k}{d}
\end{array}\right) 2^{n-\frac{2 c k}{d}}
$$

and the bound is tight if and only if, either (a) or (b) hold:

(a) When $\frac{c}{d}=1, \mathcal{A}=\{\{1, \ldots, \kappa\}\} \times 2^{Y}, \mathcal{B}=\left(\begin{array}{c}{[\kappa]} \\ k\end{array}\right)$ where $Y=\{\kappa+1, \ldots, n\}$ and $\kappa \in\{2 k-1,2 k\}$ up to a relabelling of the elements of $[n]$.

(b) When $\frac{c}{d} \neq 1$ :

(i) If $k$ is even, $c=1, d=2, \frac{c k}{d}=\left\lceil\frac{k}{2}\right\rceil$,

(ii) If $k$ is odd, $c=\frac{k+1}{2}, d=k, \frac{c k}{d}=\left\lceil\frac{k}{2}\right\rceil$,

and for both the cases(( $i)$ and $(i i))$, there exists some $\tau$ such that, $k+\tau \leq n$ and up to a relabelling of the elements of $[n]$,

$\mathcal{A}=\left\{\cup_{T \in J} T: J \subset\{\{1, k+1\}, \ldots,\{\tau, k+\tau\},\{\tau+1\}, \ldots,\{k\}\},|J|=\left\lceil\frac{k}{2}\right\rceil\right\} \times 2^{X}$

where $X=\{k+\tau+1, \ldots, n\}$ and

$$
\mathcal{B}=\{L \cup\{\tau+1, \ldots, k\}: L \subset\{1, \ldots, \tau, k+1, \ldots, k+\tau\},|L \cap\{i, k+i\}|=1 \text { for }
$$

\section{Notations and definitions}

Given any $S \subseteq[n]$, we shall use $\chi(S)$ to denote the characteristic vector of $S$ which is a $0-1$ vector of size $n$ having its $i^{\text {th }}$ entry equal to 1 if and only if $i \in S$. The weight of a vector is the number of non-zero entries it has, and hence weight of $\chi(S)$ is the same as $|S|$.

For any family $\mathcal{A} \subseteq 2^{[n]}$, we shall (ab)use $\mathcal{A}$ to denote the collection of characteristic vectors of the members of $\mathcal{A}$ as well. The meaning will be clearly stated if not clear from the context.

Let $V$ be a collection of vectors in $\mathbb{F}_{2}^{n}$. Then, we define the following: 
1. $\operatorname{span}(V)$ : The collection of all the vectors that can be expressed as a linear combination in $\mathbb{F}_{2}$ of the vectors of $V$. We know that $\operatorname{span}(V)$ is a vector space over $\mathbb{F}_{2}$.

2. $\operatorname{basis}(V)$ : We use $\operatorname{basis}(V)$ to denote the basis of $\operatorname{span}(V)$.

3. $\operatorname{dim}(V): \operatorname{dim}(V)=|\operatorname{basis}(V)|$

Definition 1. $V \subseteq \mathbb{F}_{2}^{n}$ is a linear code if $V=\operatorname{span}(V)$.

Definition 2. Given a linear code $C \subseteq \mathbb{F}_{2}^{n}$, the dual code $C^{\perp}$ is defined as,

$$
C^{\perp}=\left\{x \in \mathbb{F}_{2}^{n} \mid\langle x, c\rangle=0, \forall c \in C\right\}
$$

where $\langle x, y\rangle$ is the standard inner product over $\mathbb{F}_{2}$.

The following is a well-known fact that is easy to verify.

Lemma 2.1. If $C \subseteq \mathbb{F}_{2}^{n}$ is a linear code, then $C^{\perp}$ is also a linear code.

Definition 3. Self orthogonal and self dual codes: $A$ code $C$ is self orthogonal if $C \subseteq C^{\perp}$ and it is self dual if $C=C^{\perp}$.

\section{Bounding $\mathcal{M}_{\frac{c}{d}}(n)$}

Let $(\mathcal{A}, \mathcal{B})$ be a $\frac{c}{d}$-cross-intersecting pair of families of subsets of $[n]$, where $\frac{c}{d} \in[0,1]$ is an irreducible fraction. We shall (ab)use $\mathcal{A}, \mathcal{B}$ to denote the set of characteristic vectors of the sets in $\mathcal{A}, \mathcal{B}$ respectively. For any $a \in \mathcal{A}, b \in \mathcal{B}$, we observe that $\langle a, b\rangle \equiv|A \cap B|(\bmod 2)$, where $a=\chi(A), b=\chi(B)$.

Partition the family $\mathcal{B}$ into two parts as,

$$
\begin{aligned}
& \mathcal{B}_{1}=\{B \in \mathcal{B}:|B| \equiv 0(\bmod 2 d)\} \\
& \mathcal{B}_{2}=\{B \in \mathcal{B}:|B| \equiv d(\bmod 2 d)\}
\end{aligned}
$$

As all the sets $B \in \mathcal{B}$ have their cardinality $|B|$ divisible by $d,\left\{\mathcal{B}_{1}, \mathcal{B}_{2}\right\}$ is a valid partition of $\mathcal{B}$. Therefore $\forall a \in \mathcal{A}, b \in \mathcal{B}$, using the $\frac{c}{d}$ intersection property, we have:

$$
\langle a, b\rangle=\left\{\begin{array}{l}
1, \text { if } b \in \mathcal{B}_{2} \text { and } c \text { is odd } \\
0, \text { otherwise }
\end{array}\right.
$$


Construction 1. Construct a set $\mathcal{B}_{1}^{\prime}$, by appending a 0 to the left of every vector in $\mathcal{B}_{1}$, and a set $\mathcal{B}_{2}^{\prime}$ by appending a 1 to the left of every vector in $\mathcal{B}_{2}$. Let $\mathcal{B}^{\prime}=\mathcal{B}_{1}^{\prime} \cup \mathcal{B}_{2}^{\prime}$. Construct a set $\mathcal{A}^{\prime}$ by appending a 1 to the left of every vector in $\mathcal{A}$.

We now have, the value of

$$
\langle a, b\rangle=0 \quad \forall a \in \mathcal{A}^{\prime}, b \in \mathcal{B}^{\prime}
$$

So, $\left(\operatorname{span}\left(\mathcal{A}^{\prime}\right), \operatorname{span}\left(\mathcal{B}^{\prime}\right)\right)$ is a pair of mutually orthogonal subspaces of $\mathbb{F}_{2}^{n+1}$ over $\mathbb{F}_{2}$. We thus have,

$$
\operatorname{dim}\left(\operatorname{span}\left(\mathcal{A}^{\prime}\right)\right)+\operatorname{dim}\left(\operatorname{span}\left(\mathcal{B}^{\prime}\right)\right) \leq n+1
$$

So, it follows that

$$
\begin{aligned}
\left|\operatorname{span}\left(\mathcal{A}^{\prime}\right)\right| \cdot\left|\operatorname{span}\left(\mathcal{B}^{\prime}\right)\right| & =2^{\operatorname{dim}\left(\operatorname{span}\left(\mathcal{A}^{\prime}\right)\right)} \cdot 2^{\operatorname{dim}\left(\left(\operatorname{span}\left(\mathcal{B}^{\prime}\right)\right)\right.} \\
& =2^{\operatorname{dim}\left(\operatorname{span}\left(\mathcal{A}^{\prime}\right)\right)+\operatorname{dim}\left(\operatorname{span}\left(\mathcal{B}^{\prime}\right)\right)} \\
& \leq 2^{n+1}
\end{aligned}
$$

Lemma 3.1. If the elements of a linear code $C \subseteq \mathbb{F}_{2}^{n}$ are arranged as rows of a matrix $M_{C}$ with $n$ columns, then for each column, one of the following holds,

(i) All the entries in that column are 0

(ii) Exactly half the entries in that column are 0 , and the rest are 1.

Proof. As $C$ is a linear code, if we pick any $a \in C$, and consider the set $S=$ $\{a+x \mid x \in C\}$ where $a+x$ is the vector addition in $\mathbb{F}_{2}^{n}$, then by the definition of a linear code $S=C$. Let $M_{S}$ be a matrix whose rows are the vectors of $S$, taken in any order. $M_{S}$ and $M_{C}$ have the same set of rows (only their order may differ).

Let $j \in[n]$. Column $j$ in $M_{C}$ and $M_{S}$ have the same number of 1's( and 0's). Suppose (i) does not hold for column $j$ in $M_{C}$. Then, some row, say $a$, in $M_{C}$ has its $j^{\text {th }}$ entry as 1 . Let $S$, and thereby $M_{S}$, be defined according to this vector $a$. From the definition of $S$, it is clear that the number of 1 's in the $j^{\text {th }}$ column of $M_{S}$ is equal to the number of 1's in the $j^{\text {th }}$ column of $M_{C}$. Since adding $a$ to any $\{0,1\}$ vector flips the $j^{\text {th }}$ coordinate of $v$, we conclude that $(i i)$ holds for $M_{c}$.

Corollary 3.2. $\left|\operatorname{span}\left(\mathcal{A}^{\prime}\right)\right| \geq 2\left|\mathcal{A}^{\prime}\right|$

Proof. The leftmost column of $\mathcal{M}_{\mathcal{A}^{\prime}}$ does not contain any 0 . As $\operatorname{span}\left(\mathcal{A}^{\prime}\right)$ is a linear code and $\mathcal{A}^{\prime} \subseteq \operatorname{span}\left(\mathcal{A}^{\prime}\right)$, by condition (ii) of Lemma 3.1 above, $\operatorname{span}\left(\mathcal{A}^{\prime}\right)$ must have at least $\left|\mathcal{A}^{\prime}\right|$ more elements having their leftmost entry as 0 . 
Now we prove the main result of this section which is Theorem 1.1 .

Statement of Theorem 1.1: $\mathcal{M}_{\frac{c}{d}}(n)=2^{n}$

Proof. $\mathcal{A}=2^{[n]}, \mathcal{B}=\{\emptyset\}$ is a trivial example of a $\frac{c}{d}$ cross-intersecting pair of families having $|\mathcal{A}||\mathcal{B}|=2^{n}$. Thus, $\mathcal{M}_{\frac{c}{d}}(n) \geq 2^{n}$. The proof of the upper bound for $\mathcal{M}_{\frac{c}{d}}(n)$ follows from Inequality (3) and Corollary 3.2 . Let $(\mathcal{A}, \mathcal{B})$ be a $\frac{c}{d}$ crossintersecting pair of families of subsets of $[n]$. Let $\mathcal{A}^{\prime}, \mathcal{B}^{\prime}$ be constructed from $\mathcal{A}$, $\mathcal{B}$, respectively, as explained in the beginning of this section. Note that $\left|\mathcal{A}^{\prime}\right|=|\mathcal{A}|$ and $\left|\mathcal{B}^{\prime}\right|=|\mathcal{B}|$ by construction.

$$
\begin{aligned}
2^{n+1} & \geq\left|\operatorname{span}\left(\mathcal{A}^{\prime}\right)\right| \cdot\left|\operatorname{span}\left(\mathcal{B}^{\prime}\right)\right| & & {[\text { from }(3)] } \\
& \geq 2 \cdot\left|\mathcal{A}^{\prime}\right| \cdot\left|\operatorname{span}\left(\mathcal{B}^{\prime}\right)\right| & & {[\text { from Corollary } 3.2] } \\
& \geq 2 \cdot\left|\mathcal{A}^{\prime}\right| \cdot\left|\mathcal{B}^{\prime}\right| & & \\
& =2 \cdot|\mathcal{A}| \cdot|\mathcal{B}| & & \text { [by construction] }
\end{aligned}
$$

\section{Characterization of maximal pairs when $\frac{c}{d}=\frac{1}{2}$}

Definition 4. Cross bisecting pair of families: A pair of families of subsets of $[n]$ is called a cross-bisecting pair if it is a $\frac{1}{2}$ cross-intersecting pair. $(\mathcal{A}, \mathcal{B})$ is called a maximal cross bisecting or simply a maximal pair, if it is a cross bisecting pair and $|\mathcal{A}||\mathcal{B}|=2^{n}$.

For example, $\mathcal{A}=2^{[n]}$ and $\mathcal{B}=\{\emptyset\}$ is a trivial maximal pair. In this section, we characterize all maximal pairs. Let $(\mathcal{A}, \mathcal{B})$ be a cross bisecting pair and let $\left(\mathcal{A}^{\prime}, \mathcal{B}^{\prime}\right)$ be the associated pair constructed by appending bits as defined in the previous section.

Definition 5. Let $f_{\mathcal{A}}: \mathcal{A} \rightarrow \mathcal{A}^{\prime}$ be a bijective mapping that maps every vector in $\mathcal{A}$ to its corresponding vector in $\mathcal{A}^{\prime}$, and let $g_{\mathcal{A}}: \mathcal{A}^{\prime} \rightarrow \mathcal{A}$ be its inverse. Likewise, define functions $f_{\mathcal{B}}$ and $g_{\mathcal{B}}$ between $\mathcal{B}$ and $\mathcal{B}^{\prime}$. For any set $V \subseteq \mathcal{A}$, we shall use, $f_{\mathcal{A}}(V)$ to denote $\left\{f_{\mathcal{A}}(A) \mid A \in V\right\}$ and for any $V \subseteq \mathcal{A}^{\prime}$, we use $g_{\mathcal{A}}(V)$ to denote $\left\{g_{\mathcal{A}}(A) \mid A \in V\right\}$. Similarly, for any $V \subseteq \mathcal{B}$, we use, $f_{\mathcal{B}}(V)$ to denote $\left\{f_{\mathcal{B}}(B) \mid B \in V\right\}$ and for any $V \subseteq \mathcal{B}^{\prime}, g_{\mathcal{B}}(V)$ to denote $\left\{g_{\mathcal{B}}(B) \mid B \in V\right\}$

Observation 1. $f_{\mathcal{B}}\left(\mathcal{B}_{1}\right)=\mathcal{B}_{1}^{\prime}$ and $f_{\mathcal{B}}\left(\mathcal{B}_{2}\right)=\mathcal{B}_{2}^{\prime}$. Similarly, g $g_{\mathcal{B}}\left(\mathcal{B}_{1}^{\prime}\right)=\mathcal{B}_{1}$ and $g_{\mathcal{B}}\left(\mathcal{B}_{2}^{\prime}\right)=\mathcal{B}_{2}$ 
Suppose $(\mathcal{A}, \mathcal{B})$ is a maximal pair. Then from the proof of Theorem 1.1, we must have :

$$
\begin{aligned}
\left|\operatorname{span}\left(\mathcal{A}^{\prime}\right)\right| & =2\left|\mathcal{A}^{\prime}\right| \\
\left|\operatorname{span}\left(\mathcal{B}^{\prime}\right)\right| & =\left|\mathcal{B}^{\prime}\right| \\
\operatorname{dim}\left(\operatorname{span}\left(\mathcal{A}^{\prime}\right)\right)+\operatorname{dim}\left(\operatorname{span}\left(\mathcal{B}^{\prime}\right)\right) & =n+1
\end{aligned}
$$

Proposition 4.1. $\mathcal{B}=\operatorname{span}(\mathcal{B})$. Further, $f_{\mathcal{B}}$ is a linear map.

Proof. This follows from equation (5). Let $x_{1}, x_{2} \in \mathcal{B}$. We show that $x_{3}=x_{1}+$ $x_{2} \in \mathcal{B}$. This would imply $\mathcal{B}$ is closed under addition in $\mathbb{F}_{2}^{n}$ over $\mathbb{F}_{2}$, and hence $\mathcal{B}=\operatorname{span}(\mathcal{B})$.

Let $x_{1}^{\prime}=f_{\mathcal{B}}\left(x_{1}\right)$ and $x_{2}^{\prime}=f_{\mathcal{B}}\left(x_{2}\right)$. From Equation (5), we have, $w=x_{1}^{\prime}+x_{2}^{\prime} \in$ $\mathcal{B}^{\prime}$. Since $w$ and $x_{3}$ agree on each of the rightmost $n$ bits of $x_{3}$, we have $g_{\mathcal{B}}(w)=x_{3}$. Since $w \in \mathcal{B}^{\prime}$, from the definition of the function $g_{\mathcal{B}}$ we have $x_{3}=g_{\mathcal{B}}(w) \in \mathcal{B}$. Further, observe that $f_{\mathcal{B}}\left(x_{1}\right)+f_{\mathcal{B}}\left(x_{2}\right)=w=f_{\mathcal{B}}\left(x_{3}\right)=f_{\mathcal{B}}\left(x_{1}+x_{2}\right)$ and hence $f_{\mathcal{B}}$ is a linear map.

That $\mathcal{B}$ is a linear code from Proposition 4.1 implies closure of the family of subsets $\mathcal{B}$ under symmetric difference. In fact, we have the following stronger result.

Proposition 4.2. Let vectors $b_{1}, b_{2} \in \mathcal{B}$. Then, $b_{1}+b_{2} \in \mathcal{B}_{1}$ if and only if either $b_{1}, b_{2} \in \mathcal{B}_{1}$, or $b_{1}, b_{2} \in \mathcal{B}_{2}$. Otherwise, $b_{1}+b_{2} \in \mathcal{B}_{2}$.

Proof. We prove the 2-way implication, and rest of the proposition follows from Proposition 4.1. Let $b_{1}^{\prime}=f_{\mathcal{B}}\left(b_{1}\right), b_{2}^{\prime}=f_{\mathcal{B}}\left(b_{2}\right)$.

- $b_{1}+b_{2} \in \mathcal{B}_{1} \Rightarrow b_{1}$ and $b_{2}$ are both from $\mathcal{B}_{1}$, or both from $\mathcal{B}_{2}$

Since $f_{\mathcal{B}}$ is a linear map, we have $\left(b_{1}+b_{2} \in \mathcal{B}_{1}\right) \Rightarrow\left(f_{\mathcal{B}}\left(b_{1}+b_{2}\right)=f_{\mathcal{B}}\left(b_{1}\right)+\right.$ $\left.f_{\mathcal{B}}\left(b_{2}\right)=b_{1}^{\prime}+b_{2}^{\prime} \in \mathcal{B}_{1}^{\prime}\right)$. So, the leftmost bit of $b_{1}^{\prime}+b_{2}^{\prime}$ is 0 . This means that the leftmost bit must be the same in $b_{1}^{\prime}$ and $b_{2}^{\prime}$, which directly implies that either $b_{1}^{\prime}, b_{2}^{\prime} \in \mathcal{B}_{1}^{\prime}$, or $b_{1}^{\prime}, b_{2}^{\prime} \in \mathcal{B}_{2}^{\prime}$.

- Either $b_{1}, b_{2} \in \mathcal{B}_{1}$, or $b_{1}, b_{2} \in \mathcal{B}_{2} \Rightarrow b_{1}+b_{2} \in \mathcal{B}_{1}$

Since $b_{1}^{\prime}$ and $b_{2}^{\prime}$ agree upon the leftmost bit, $b_{1}^{\prime}+b_{2}^{\prime}$ has a 0 in its leftmost bit. So, $b_{1}^{\prime}+b_{2}^{\prime} \in \mathcal{B}_{1}^{\prime}$. From the Observation 1 above, we have $b_{1}+b_{2} \in \mathcal{B}_{1}$.

Proposition 4.3. $\mathcal{B}$ is a self-orthogonal code. 
Proof. We prove the proposition by showing that $\forall b_{1}, b_{2} \in \mathcal{B},\left\langle b_{1}, b_{2}\right\rangle=0$. Let $B_{1}, B_{2}$ be the sets corresponding to the vectors $b_{1}, b_{2}$, respectively. Since we are operating in the field $\mathbb{F}_{2}$, it is enough to show that $\left|B_{1} \cap B_{2}\right|$ is even.

Let $b_{3}=b_{1}+b_{2}$. We observe that $b_{3}$ is the characteristic vector of $B_{3}=B_{1} \Delta B_{2}$, the symmetric difference of $B_{1}$ and $B_{2}$. We have,

$$
\left|B_{3}\right|=\left|B_{1} \Delta B_{2}\right|=\left|B_{1}\right|+\left|B_{2}\right|-2\left|B_{1} \cap B_{2}\right|
$$

As $\frac{c}{d}=\frac{1}{2}, \forall B \in \mathcal{B}_{1}$, we have $|B| \equiv 0(\bmod 4)$. By Proposition $4.1, B_{1} \Delta B_{2}=$ $B_{3} \in \mathcal{B}$ as $\mathcal{B}$ is a linear code. Taking equation (7) modulo 4 , if $B_{3} \in \mathcal{B}_{1}$, then

$$
\left|B_{1}\right|+\left|B_{2}\right|-2\left|B_{1} \cap B_{2}\right| \equiv 0(\bmod 4)
$$

By Proposition 4.2 , both $B_{1}$ and $B_{2}$ are either from $\mathcal{B}_{1}$ or from $\mathcal{B}_{2}$. In both cases, $\left|B_{1}\right|+\left|B_{2}\right| \equiv 0(\bmod 4)$ Therefore, $2\left|B_{1} \cap B_{2}\right| \equiv 0(\bmod 4)$ or $\left|B_{1} \cap B_{2}\right| \equiv 0(\bmod 2)$. If $B_{3} \in \mathcal{B}_{2}$, then

$$
\left|B_{1}\right|+\left|B_{2}\right|-2\left|B_{1} \cap B_{2}\right| \equiv\left|B_{3}\right| \equiv 2(\bmod 4)
$$

Again by Proposition 4.2, $\left|B_{1}\right|+\left|B_{2}\right| \equiv 2(\bmod 4)$.

So, we have $2\left|B_{1} \cap B_{2}\right| \equiv 0(\bmod 4)$ or $\left|B_{1} \cap B_{2}\right| \equiv 0(\bmod 2)$. Thus in both cases, $\left|B_{1} \cap B_{2}\right|$ is even, so $\mathcal{B}$ is a self-othogonal code.

Lemma 4.4. Let $(\mathcal{A}, \mathcal{B})$ be a maximal pair, then $|\mathcal{B}| \leq 2^{\left\lfloor\frac{n}{2}\right\rfloor}$

Proof. It is a known result (see [11]) that for a linear code $C \subseteq \mathbb{F}_{2}^{n}$ and its dual code $C^{\perp}$,

$$
\operatorname{dim}(C)+\operatorname{dim}\left(C^{\perp}\right)=n
$$

For any self-orthogonal code $C, C \subseteq C^{\perp}$. So,

$$
\operatorname{dim}(C) \leq \operatorname{dim}\left(C^{\perp}\right)
$$

Applying equation (8) in this inequality, we get

$$
\begin{gathered}
n=\operatorname{dim}(C)+\operatorname{dim}\left(C^{\perp}\right) \geq 2 \operatorname{dim}(C) \\
\text { Therefore, } \operatorname{dim}(C) \leq \frac{n}{2}
\end{gathered}
$$

Since $\mathcal{B}$ is a self-orthogonal code (Proposition 4.3), we get $\operatorname{dim}(\mathcal{B}) \leq \frac{n}{2}$. Hence,

$$
|\mathcal{B}| \leq 2^{\left\lfloor\frac{n}{2}\right\rfloor}
$$


Proposition 4.5. If a set $A$ bisects $B_{1}, B_{2}$ and $B_{1} \Delta B_{2}$, then $A$ also bisects $B_{1} \cap B_{2}$.

Proof.

$$
\begin{aligned}
\left|A \cap\left(B_{1} \triangle B_{2}\right)\right| & =\frac{\left|B_{1} \Delta B_{2}\right|}{2}\left[\text { A bisects } B_{1} \Delta B_{2}\right] \\
\Rightarrow\left|A \cap\left(\left(B_{1} \backslash B_{2}\right) \cup\left(B_{2} \backslash B_{1}\right)\right)\right| & =\frac{\left|B_{1}\right|+\left|B_{2}\right|-2\left|B_{1} \cap B_{2}\right|}{2} \\
\Rightarrow\left|A \cap\left(B_{1} \backslash B_{2}\right)\right|+\left|A \cap\left(B_{2} \backslash B_{1}\right)\right| & =\frac{\left|B_{1}\right|}{2}+\frac{\left|B_{2}\right|}{2}-\left|B_{1} \cap B_{2}\right| \\
\Rightarrow\left|A \cap B_{1}\right|-\left|A \cap\left(B_{1} \cap B_{2}\right)\right|+\left|A \cap\left(B_{2}\right)\right| & -\left|A \cap\left(B_{1} \cap B_{2}\right)\right|=\frac{\left|B_{1}\right|}{2}+\frac{\left|B_{2}\right|}{2}-\left|B_{1} \cap B_{2}\right| \\
\Rightarrow \frac{\left|B_{1}\right|}{2}+\frac{\left|B_{2}\right|}{2}-2\left|A \cap\left(B_{1} \cap B_{2}\right)\right| & =\frac{\left|B_{1}\right|}{2}+\frac{\left|B_{2}\right|}{2}-\left|B_{1} \cap B_{2}\right| \\
\Rightarrow 2\left|A \cap\left(B_{1} \cap B_{2}\right)\right| & =\mid \frac{\left|B_{1} \cap B_{2}\right|}{2} \\
\Rightarrow\left|A \cap\left(B_{1} \cap B_{2}\right)\right| & =\frac{\left|B_{1} \cap B_{2}\right|}{2}
\end{aligned}
$$

Proposition 4.6. $\mathcal{B}$ is closed under intersection.

Proof. Let $B_{1}, B_{2} \in \mathcal{B}$. We show that $B_{1} \cap B_{2} \in \mathcal{B}$. By Proposition 4.1, $b_{1}+b_{2} \in \mathcal{B}$ i.e., $B_{1} \Delta B_{2} \in \mathcal{B}$. Let $A$ be any arbitrary member of $\mathcal{A}$. Now, $A$ bisects $B_{1}, B_{2}$ and $B_{1} \Delta B_{2}$ as $(\mathcal{A}, \mathcal{B})$ is a cross bisecting pair. By Proposition 4.5, $A$ bisects $B_{1} \cap B_{2}$. Since $(\mathcal{A}, \mathcal{B})$ is a maximal pair, we conclude that $B_{1} \cap B_{2} \in \mathcal{B}$.

Now, we prove the main result of this section, Theorem 1.2 , the characterization of maximal pairs.

Statement of Theorem 1.2: Let $(\mathcal{A}, \mathcal{B})$ be a $\frac{1}{2}$-cross intersecting pair of families of subsets of $[n]$ with $|\mathcal{A}||\mathcal{B}|=2^{n}$. Then $(\mathcal{A}, \mathcal{B})$ is one of the following $\left\lfloor\frac{n}{2}\right\rfloor+1$ pairs of families $\left(\mathcal{A}_{k}, \mathcal{B}_{k}\right), 0 \leq k \leq\left\lfloor\frac{n}{2}\right\rfloor$, up to isomorphism.

$$
\begin{gathered}
\mathcal{A}_{0}=2^{[n]} \text { and } \mathcal{B}_{0}=\{\emptyset\} \\
\mathcal{A}_{k}=\left\{A \in 2^{[n]}:|A \cap\{2 i-1,2 i\}|=1 \quad \forall i, 1 \leq i \leq k\right\} \\
\mathcal{B}_{k}=\left\{B \in 2^{[n]}:|B \cap\{2 i-1,2 i\}| \in\{0,2\} \quad \forall i, 1 \leq i \leq k \text { and } \forall j>2 k, j \notin B\right\},
\end{gathered}
$$


where $1 \leq k \leq\left\lfloor\frac{n}{2}\right\rfloor$.

By isomorphism, it is meant that for any maximal pair $(\mathcal{A}, \mathcal{B}), \exists$ a bijective mapping $f:[n] \rightarrow[n]$ such that if every $A \in \mathcal{A}$ is replaced by $A_{f}=\{f(i) \mid i \in A\}$ and every $B \in \mathcal{B}$ is replaced by $B_{f}=\{f(i) \mid i \in B\}$ then the families $\left(\mathcal{A}_{f}, \mathcal{B}_{f}\right)$, where $\mathcal{A}_{f}=\left\{A_{f} \mid A \in \mathcal{A}\right\}$ and $\mathcal{B}_{f}=\left\{B_{f} \mid B \in \mathcal{B}\right\}$, is a maximal pair which is one of $\left(\mathcal{A}_{k}, \mathcal{B}_{k}\right), 0 \leq k \leq\left\lfloor\frac{n}{2}\right\rfloor$.

Proof. Consider a maximal pair $(\mathcal{A}, \mathcal{B})$ where $\mathcal{B} \neq\{\emptyset\}$. We write the elements of $\mathcal{B}$ as rows of a $0-1$ matrix $M_{0}$. Suppose $n_{0}$ columns have only 0 entries in all the rows $\left(n_{0}\right.$ may be 0$)$. As the characterization is up to isomorphism, we may assume that these are the rightmost $n_{0}$ columns of the matrix $M_{0}$. In each of the remaining $n-n_{0}$ columns, from Lemma 3.1, there are exactly $\frac{|\mathcal{B}|}{2} 1$ 's and $\frac{|\mathcal{B}|}{2} 0$ 's as $\mathcal{B}$ is a linear code. (by Proposition 4.1 )

Define

$$
B_{1}=\bigcap_{\substack{1 \in B, B \in \mathcal{B}}} B
$$

We write the $\frac{|\mathcal{B}|}{2}$ rows containing 1 in the leftmost column of $M_{0}$ as the top $\frac{|\mathcal{B}|}{2}$ rows to obtain a new matrix $M_{1}$ from $M_{0}$. And $B_{1}$ is one of these rows according to Proposition 4.6. Moreover, as all intersections are of even cardinality (Proposition $4.3,\left|B_{1}\right|$ is even.

Let $\left|B_{1}\right|=2 i_{1}, i_{1} \geq 1$. So, there are $2 i_{1}-1$ elements in $B_{1}$ other than the element 1. Due to isomorphism, we may assume them to be $2,3, \ldots, 2 i_{1}$. If $2 i_{1}+1 \leq n-n_{0}$, then define the set $B_{2}$ as:

$$
B_{2}=\bigcap_{\substack{2 i_{1}+1 \in B, B \in \mathcal{B}}} B
$$

Claim 4.7. $1 \notin B_{2}$

Proof. Assume for the sake of contradiction, $1 \in B_{2}$. This implies that for all the $\frac{|\mathcal{B}|}{2}$ sets which contain the element $2 i_{1}+1$ also contain the element 1 . From Lemma 3.1. (number of sets in $\mathcal{B}$ that contain the element 1$)=($ number of sets in $\mathcal{B}$ that contain the element $\left.2 i_{1}+1\right)=\frac{|\mathcal{B}|}{2}$. Hence, for any $B \in \mathcal{B}, 1 \in B \Longleftrightarrow$ $2 i_{1}+1 \in B$. This implies that $2 i_{1}+1 \in B_{1}$, which is a contradiction. Hence, $1 \notin B_{2}$ and therefore $B_{2}$ does not belong to the top $\frac{|\mathcal{B}|}{2}$ rows of $M_{1}$.

Claim 4.8. $B_{1} \cap B_{2}=\emptyset$

Proof. Assume for the sake of contradiction, $x \in B_{1} \cap B_{2}$. Then $x$ is present in the $\frac{|\mathcal{B}|}{2}$ rows of the matrix $M_{1}$ whose intersection yields $B_{1}$. Since $x \in B_{2}$ and $B_{2}$ does not belong to these $\frac{|\mathcal{B}|}{2}$ rows of $M_{1}$ (by Claim 4.7). Thus, we have the element $x$ present in at least $\frac{|\mathcal{B}|}{2}+1$ rows of $M_{1}$, contradicting Lemma 3.1 . 
We take the rows corresponding to the sets containing the $\left(2 i_{1}+1\right)^{t h}$ element that are not among the first $\frac{|\mathcal{B}|}{2}$ rows in $M_{1}$ and arrange them below the top $\frac{|\mathcal{B}|}{2}$ rows to create a matrix called $M_{2}$ from $M_{1}$. Again from Proposition $4.3,\left|B_{2}\right|$ is even, say $2 i_{2}$. Due to isomorphism and Claim 4.8 , we may assume that $2 i_{1}+1, \ldots, 2 i_{1}+2 i_{2}$ are these $2 i_{2}$ elements.

If $2 i_{1}+2 i_{2}+1 \leq n-n_{0}$, then define,

$$
B_{3}=\bigcap_{\substack{2 i_{1}+2 i_{2}+\mathcal{B} \in B, B \in \mathcal{B}}} B
$$

Claim 4.9. $1 \notin B_{3}$ and $2 i_{1}+1 \notin B_{3}$.

The proof is similar to that of Claim 4.7

Claim 4.10. $B_{1} \cap B_{3}=\emptyset$ and $B_{2} \cap B_{3}=\emptyset$.

The proof is again similar to that of Claim 4.8 .

We take the rows corresponding to the sets containing the $\left(2 i_{1}+2 i_{2}+1\right)^{t h}$ element that are not among the first $r$ rows $\left(r>\frac{|\mathcal{B}|}{2}\right)$ in $M_{2}$ which contain the elements 1 or $2 i_{1}+1$ and arrange them below the top $r$ rows of $M_{2}$ to create a matrix called $M_{3}$ from $M_{2}$. From Proposition 4.3 and the definition of $B_{3}$, we have $\left|B_{3}\right|=2 i_{3}, i_{3} \geq 1$. Due to isomorphism and Claim 4.10 , we may assume that $2 i_{1}+2 i_{2}+1, \ldots, 2 i_{1}+2 i_{2}+2 i_{3}$ are these $2 i_{3}$ elements.

We continue in this manner for $k$ steps by constructing sets $B_{1}, \ldots, B_{k}$ and matrices $M_{1}, \ldots, M_{k}$, where $k \geq 1$, until we have $2 i_{1}+\cdots+2 i_{k}=n-n_{0}$. Observe that $B_{1}, \ldots, B_{k}$ and $P=\left\{n-n_{0}+1, \ldots, n\right\}$ is a partition of $[n]$.

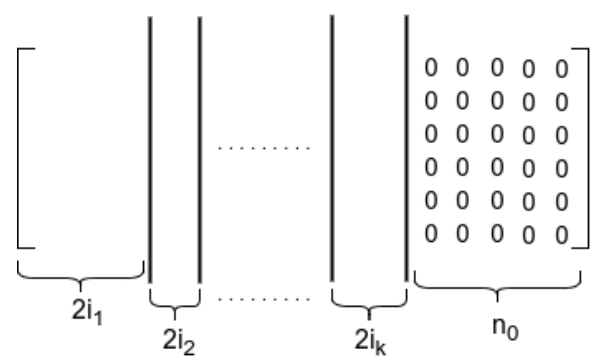

Figure 1: Partitioning the universe and thereby the columns of $M_{k}$

Claim 4.11. For any set $B \in \mathcal{B}, j \in[k]$, we have $B \cap B_{j} \in\left\{\emptyset, B_{j}\right\}$. Further, $B \cap P=\emptyset$.

Proof. From the definition of $P$, we have $B \cap P=\emptyset$. Let $j \in[k]$. Since $B_{j}$ is equal to the intersection of some $\frac{|\mathcal{B}|}{2}$ sets in $\mathcal{B}$, we have $B_{j}$ present as a subset of all these $\frac{|\mathcal{B}|}{2}$ sets. Applying Lemma 3.1, we can say that no element of $B_{j}$ is present in any set in $\mathcal{B}$ other than these $\frac{|\mathcal{B}|}{2}$ sets. Hence, the claim. 
From Claim 4.11, observe that $S=\left\{B_{1}, \ldots, B_{k}\right\}$ forms a basis of the row space of the matrix $M_{k}$. The advantage of such a "disjoint basis" is that the bisection in one part is independent of another.

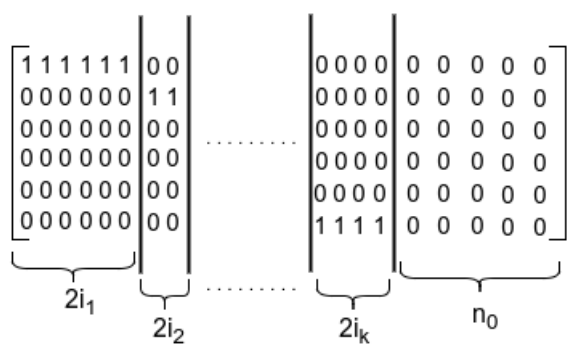

Figure 2: Basis for the code $\mathcal{B}$

Claim 4.12. $A$ set $A \in \mathcal{A}$ bisects every set in $\mathcal{B}$ if and only if it bisects every set in the basis $S$ of $\mathcal{B}$.

Proof. The forward direction is straightforward as $S \subseteq \mathcal{B}$. For the opposite direction, let $A \in \mathcal{A}$ be a set that bisects every member of $S$. Since the sets corresponding to the members in $S$ are disjoint, any $B \in \mathcal{B}$ can be written as a union of some of these sets.

Let $B=B_{1} \cup \cdots \cup B_{l}$, where $\left\{B_{1}, \ldots, B_{l}\right\} \subseteq S$. Then,

$$
|A \cap B|=\left|A \cap\left(\bigcup_{j=1}^{l} B_{j}\right)\right|=\sum_{j=1}^{l}\left|A \cap B_{j}\right|=\sum_{j=1}^{l} \frac{\left|B_{j}\right|}{2}=\frac{\left|\bigcup_{j=1}^{l} B_{j}\right|}{2}=\frac{|B|}{2}
$$

Since each set $A \in \mathcal{A}$ bisects the sets $B_{1}, \ldots, B_{k}$ and $P$, from Claim 4.12 , the set $A$ may contain any of the $2^{n_{0}}$ subsets of $P$, and $\left|A \cap B_{1}\right|=i_{1}, \ldots,\left|A \cap B_{k}\right|=i_{k}$. Since $\operatorname{dim}(\mathcal{B})=k$, by Proposition 4.1 , we have $|\mathcal{B}|=2^{k}$.

$$
|\mathcal{A}||\mathcal{B}|=\left(2^{n_{0}} \cdot \prod_{j=1}^{k}\left(\begin{array}{c}
2 i_{j} \\
i_{j}
\end{array}\right)\right) \cdot 2^{k}
$$

Recall that $\sum_{j=1}^{k} 2 i_{j}=n-n_{0}$. Right hand side of Equation (9), is equal to $2^{n}$ if and only if $i_{j}=1, \forall j \in[k]$.

Thus, if $\mathcal{B} \neq\{\emptyset\}$, then $\left(\mathcal{A}_{k}, \mathcal{B}_{k}\right), k \geq 1$, defined in the statement of the theorem are the only maximal pairs. This completes the proof of Theorem 1.2 . 


\section{$5 \quad$ Tight upper bound on $M_{\frac{c}{d}}(n)$ when $\mathcal{B}$ is $k$-uniform and characterization of the cases when the bound is achieved}

Let $(\mathcal{A}, \mathcal{B})$ be a $\frac{c}{d}$ cross-intersecting pair of families of subsets of $[n]$, where $\frac{c}{d} \in[0,1]$ is an irreducible fraction. In this section, we deal with the scenario when $\mathcal{B}$ is $k$ uniform, where $0<k \leq n$. Since $\mathcal{B}$ is $k$-uniform, for any $A \in \mathcal{A}$ and any $B \in \mathcal{B}$, $|A \cap B|=\frac{c k}{d}=l$. Since $c$ is relatively prime with $d$, and $|A \cap B|$ is an integer, we have $k$ divisible by $d$. Therefore, we have a uniformly cross intersecting pair of families.

Alon and Lubetzky in [10] found a tight upper bound for the case of uniformly cross intersecting families and fully characterized the cases when the bound is achieved in the following theorem:

Theorem 5.1. [Theorem 1.1 in [10]] There exists some $l_{0}>0$ such that, for all $l \geq l_{0}$, every l-cross intersecting pair $\mathcal{A}, \mathcal{B} \subset 2^{[n]}$ satisfies:

$$
|\mathcal{A}||\mathcal{B}| \leq\left(\begin{array}{c}
2 l \\
l
\end{array}\right) 2^{n-2 l}
$$

Furthermore, if $|\mathcal{A}||\mathcal{B}|=\left(\begin{array}{c}2 l \\ l\end{array}\right) 2^{n-2 l}$, then there exists some choice of parameters $\kappa, \tau, n^{\prime}$ :

$$
\begin{gathered}
\kappa \in\{2 l-1,2 l\}, \tau \in\{0, \cdots, \kappa\} \\
\kappa+\tau \leq n^{\prime} \leq n
\end{gathered}
$$

such that upto a relabelling of the elements of $[n]$ and swapping $\mathcal{A}, \mathcal{B}$, the following holds:

$$
\begin{gathered}
\mathcal{A}=\left\{\bigcup_{T \in J} T: J \subset\{\{1, \kappa+1\}, \cdots,\{\tau, \kappa+\tau\},\{\tau+1\}, \cdots,\{\kappa\}\},|J|=l\right\} \times 2^{X}, \\
\mathcal{B}=\{L \cup\{\tau+1, \cdots, \kappa\}: L \subset\{1, \cdots, \tau, \kappa+1, \cdots, \kappa+\tau\},|L \cap\{i, \kappa+i\}|=1 \text { for } \\
\text { all } i \in[\tau]\} \times 2^{Y}
\end{gathered}
$$

where $X=\left\{\kappa+\tau+1, \cdots, n^{\prime}\right\}$ and $Y=\left\{n^{\prime}+1, \cdots, n\right\}$.

Let $(\mathcal{A}, \mathcal{B})$ be a $\frac{c}{d}$ cross-intersecting family where $\mathcal{B}$ is $k$-uniform. From Theorem 5.1. there exists a $k_{0}>0$ such that if $\frac{c k}{d}=l>k_{0}$, then $|\mathcal{A}||\mathcal{B}| \leq\left(\begin{array}{c}2 l \\ l\end{array}\right) 2^{n-2 l}$. Consider the case when $\mathcal{B}$ corresponds to $\mathcal{B}$ of Theorem 5.1. If $|\mathcal{A}||\mathcal{B}|=\left(\begin{array}{c}2 l \\ l\end{array}\right) 2^{n-2 l}$, then $n^{\prime}=n, Y=\emptyset$, and $k=\kappa$ in the statement of Theorem 5.1. Since $l=\frac{c k}{d}$ and $k \in\left\{\frac{2 c k}{d}-1, \frac{2 c k}{d}\right\}$, we have the following two cases:

Case 1: $k=\frac{2 c k}{d}-1$. Then, $(k+1) d=2 c k$. Since $\operatorname{gcd}(c, d)=1$ and $\operatorname{gcd}(k, k+1)=$ 1 , we have $k|d| 2 k$. Thus, $d=k$ or $d=2 k$. We claim that $d=2 k$ is an invalid case. 
This is because, when $d=2 k$, we have $c=k+1$. Since $\operatorname{gcd}(c, d)=1, k$ cannot be odd. And if $k$ is even, then $l=\frac{c k}{d}=\frac{k+1}{2}$ is not an integer. So, the only valid case is $d=k, c=\frac{k+1}{2}=l$ and $k$ is an odd integer.

Case 2: $k=\frac{2 c k}{d}$. Then, $\frac{c}{d}=\frac{1}{2}$, that is $(\mathcal{A}, \mathcal{B})$ is a cross bisecting pair. Since $l=\frac{c k}{d}=\frac{k}{2}$ is an integer, $k$ must be even in this case.

If $\mathcal{B}$ corresponds to $\mathcal{A}$ of Theorem 5.1, we have $X=\emptyset, \tau=0, \mathcal{B}$ is $k(=l)$-uniform, $l=\frac{c k}{d}$. Thus, we have $\frac{c}{d}=1, \mathcal{A}=\{\{1, \ldots, \kappa\}\} \times 2^{Y}$ where $Y=\{\kappa+1, \ldots, n\}$ and $\mathcal{B}=\left(\begin{array}{c}{[\kappa]} \\ k\end{array}\right), \kappa \in\{2 k-1,2 k\}$ up to a relabelling of the elements.

This leads us to the main result of this section.

Statement of Theorem 1.3; Let $(\mathcal{A}, \mathcal{B})$ be a $\frac{c}{d}$-cross intersecting pair of families of subsets of $[n]$. Let $\mathcal{B}$ be $k$-uniform. Then, there exists some $k_{0}>0$, such that for $k>k_{0}$ we have

$$
|\mathcal{A}||\mathcal{B}| \leq\left(\begin{array}{l}
\frac{2 c k}{d} \\
\frac{c k}{d}
\end{array}\right) 2^{n-\frac{2 c k}{d}}
$$

and the bound is tight if and only if, either $(a)$ or $(b)$ hold:

(a) When $\frac{c}{d}=1, \mathcal{A}=\{\{1, \ldots, \kappa\}\} \times 2^{Y}, \mathcal{B}=\left(\begin{array}{c}{[\kappa]} \\ k\end{array}\right)$ where $Y=\{\kappa+1, \ldots, n\}$ and $\kappa \in\{2 k-1,2 k\}$ up to a relabelling of the elements of $[n]$.

(b) When $\frac{c}{d} \neq 1$ :

(i) If $k$ is even, $c=1, d=2, \frac{c k}{d}=\left\lceil\frac{k}{2}\right\rceil$,

(ii) If $k$ is odd, $c=\frac{k+1}{2}, d=k, \frac{c k}{d}=\left\lceil\frac{k}{2}\right\rceil$,

and for both the cases(( $i)$ and (ii)), there exists some $\tau$ such that, $k+\tau \leq n$ and up to a relabelling of the elements of $[n]$,

$\mathcal{A}=\left\{\cup_{T \in J} T: J \subset\{\{1, k+1\}, \ldots,\{\tau, k+\tau\},\{\tau+1\}, \ldots,\{k\}\},|J|=\left\lceil\frac{k}{2}\right\rceil\right\} \times 2^{X}$

where $X=\{k+\tau+1, \ldots, n\}$ and

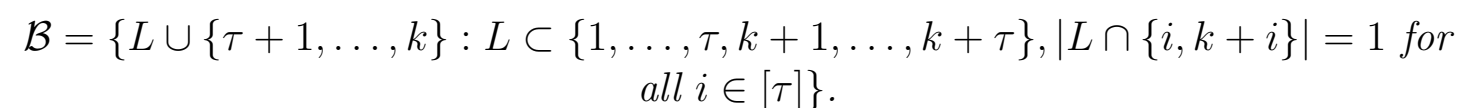

\section{Discussion}

What are those pairs of $\frac{c}{d}$-cross intersecting families $(\mathcal{A}, \mathcal{B})$ which achieve $|\mathcal{A}||\mathcal{B}|=$ $2^{n}$ (equal to the upper bound for $\mathcal{M}_{\frac{c}{d}}(n)$ proved in Theorem 1.1)? In the introduction we characterize such families when $\frac{c}{d}=0$ and $\frac{c}{d}=1$. In Theorem 1.2 , we 
characterize such families when $\frac{c}{d}=\frac{1}{2}$. From Theorem 1.3. we see that when $\mathcal{B}$ is $k$-uniform, $|\mathcal{A}||\mathcal{B}|$ is maximized when $\frac{c}{d}$ is 1 or nearly $\frac{1}{2}\left(\frac{1}{2}\right.$ or $\left.\frac{1}{2}+\frac{1}{2 k}\right)$. For $\frac{c}{d} \in(0,1)$, besides the case $\mathcal{A}=2^{[n]}, \mathcal{B}=\{\emptyset\}$, is $|\mathcal{A}||\mathcal{B}|=2^{n}$ achieved only when $\frac{c}{d}$ is close to $\frac{1}{2}$ ?

\section{References}

[1] P. Erdős, C. Ko, and R. Rado, "Intersection theorems for systems of finite sets," The Quarterly Journal of Mathematics, vol. 12, pp. 313-320, 011961.

[2] D. K. Ray-Chaudhuri and R. M. Wilson, "On t-designs," Osaka J. Math., vol. 12 , no. 3, pp. 737-744, 1975.

[3] P. Frankl and R. M. Wilson, "Intersection theorems with geometric consequences," Combinatorica, vol. 1, pp. 357-368, 121981.

[4] R. Bose et al., "A note on Fisher's inequality for balanced incomplete block designs," The Annals of Mathematical Statistics, vol. 20, no. 4, pp. 619-620, 1949 .

[5] N. Balachandran, R. Mathew, and T. K. Mishra, "Fractional L-intersecting families," CoRR, vol. abs/1803.03954, 2018.

[6] J. Liu and W. Yang, "Set systems with restricted $k$-wise $L$-intersections modulo a prime number," European Journal of Combinatorics, vol. 36, pp. 707719, 022014.

[7] L. Pyber, "A new generalization of the Erdős-Ko-Rado theorem," Journal of Combinatorial Theory, Series A, vol. 43, no. 1, pp. 85 - 90, 1986.

[8] P. Frankl, S. J. Lee, M. Siggers, and N. Tokushige, "An Erdős-Ko-Rado theorem for cross t-intersecting families," Journal of Combinatorial Theory, Series A, vol. 128, pp. $207-249,2014$.

[9] R. Ahlswede, N. Cai, and Z. Zhang, "A general 4-words inequality with consequences for 2-way communication complexity," Advances in Applied Mathematics, vol. 10, no. 1, pp. 75 - 94, 1989.

[10] N. Alon and E. Lubetzky, "Uniformly cross intersecting families," Combinatorica, vol. 29, pp. 389-431, Jul 2009.

[11] J. H. van Lint, Linear Codes, pp. 33-46. Berlin, Heidelberg: Springer Berlin Heidelberg, 1999. 